\title{
Role of the Kidney in the Development and Maintenance of Hypertension Caused by Renal Segmental Infarction in the Rat
}

\author{
Kazuki Kawabe, M.D., Kumiko Shiono, B.P., Susumu \\ Mizogami, B.P., and Hirofumi Sokabe, M.D.
}

\begin{abstract}
Summary
Utilizing the microsurgical method of transplantation in the rat, the role of the kidney in an experimental renal hypertension due to segmental renal infarction was studied. When the infarcted kidney was transplanted into the bilaterally nephrectomized recipient, hypertension developed so long as the donor kidney was transplanted within 2 weeks after infarction. With transplantation of the normal kidney into the hypertensive rat 8 weeks after infarction, BP remained high unless the infarcted kidney was removed. PRA after transplantation, or KRA of the transplanted kidneys were not correlated to the blood pressure levels. It was suggested that the infarcted kidncy has the pressor mechanism, lasting for 2 weeks or more after infarction. The infarcted kidney also has the maintenance mechanism, establishing 8 weeks after infarction.
\end{abstract}

\section{Additional Indexing Words :}

Renal transplantation Plasma renin activity Kidney renin content

$\mathrm{T}$

HE segmental infarction of kidney has been used as a means of experimental renal hypertension. But the specific role of the infarcted kidney in hypertension is still obscure.

Kawabe, Merrill, and Oken ${ }^{1)}$ suggested 2 roles of the kidney in the pathogenesis of this type of hypertension, utilizing the renal transplantation technique in the rat. They stated that partially infarcted kidney was able to elevate the blood pressure of the recipient so long as the kidney was transplanted within 12 days after infarction, and this pressor activity was followed by the blood pressure maintaining ability of the kidney, which, by itself, failed to induce hypertension in isogeneic recipients.

Present study is an extension of the previous one, aiming to elucidate more exactly the roles of the kidney in relation to plasma renin activity and renin content of the transplanted kidney.

From the Section of Urology, Sanraku Hospital, and Departments of Pharmacology, Toho University School of Medicine and Jichi Medical School.

Address for reprint request: Kazuki Kawabe, M.D., Section of Urology, Sanraku Hospital, 2-5 Kandasurugadai, Chiyoda-ku, Tokyo 101.

Received for publication January 30, 1976. 


\section{Materials and Methods}

In this study, male inbred Wistar rats (F40, Hoshino Experimental Animals, Co) were used. They were fed with regular laboratory chow and tap water ad lib. throughout the experiment. Rats weighing between 200 to $400 \mathrm{Gm}$ were used for surgery.

Blood pressure: Blood pressurc (BP) of rats was determined by the plethysmographic tail method. Measurements were made in triplicate to the nearest 10 mmHg. BP of animals was determined weekly for 8 weeks post-operatively by a different person from the transplant surgeon, avoiding the bias. BP of normal rats used in this experiment was $120 \mathrm{mmHg}$ with a few exception, and hypertension was arbitrarily defined as the tail BP over $150 \mathrm{mmHg}$.

Infarction: Partial infarction of the kidney was produced by ligating the bigger branch of the bifurcated artery at the right renal hilus through a right flank incision under ether anesthesia. The left kidney was removed through a left flank incision. Rats were used 1, 2, 4, and 8 weeks after the infarction.

Transplantation: Renal transplantation was performed with the rats under ether anesthesia, utilizing a modified microsurgical technique of Fisher and Lee, ${ }^{2}$ and Lee. ${ }^{3)}$ All anastomoses were preformed with 7-0 siliconized silk with atraumatic needle (Davis and Geck 1282-03). Recipients' own kidneys were removed or left in situ according to the protocol. The total ischemia time varied between 30 to $60 \mathrm{~min}$. Preparation of donor kidney took longer time when infarcted kidney was used for grafting because of previous operative scar. Animals were housed individually in the metabolism cages and urine output was monitored every day. The recipients, dying or failing to produce urine in the succeeding 8 weeks, were considered technical failures and excluded from the evaluation.

Renin activity: Plasma renin activity (PRA) was determined by the modified method of Boucher et al. ${ }^{41} \quad$ Blood was collected through polyethylene tube inserted into carotid artery under ether anesthesia at the termination of experiments. To prevent the excessive renin release from the kidney during manipulation, the renal pedicle was clamped prior to blood collection. Then kidneys were removed to determine the renin content (KRA) by the method of Nishimura and Sokabe. ${ }^{5}$ Renin activity was expressed as the first order reaction constant, $\mathrm{K}$, calculated from the equation:

$$
\mathrm{K}=\left\{2-\log _{10}[100(\mathrm{M}-\mathrm{X}) / \mathrm{M}]\right\} 2.3 / \mathrm{t}
$$

where $M$ is the angiotensinogen content of the plasma and $\mathrm{X}$ is the angiotensin formed during an incubation time, $t$.

Protocols: Three different protocols were used:

1) $\mathrm{I} \rightarrow \mathrm{N}$ The partially infarcted kidney was transplanted into the normal isogeneic recipient. Bilateral nephrectomy was performed at the time of transplantation.

2) $\mathrm{N} \rightarrow \mathrm{I}$ The normal right kideny was transplanted into the hypertensive recipient. The infarcted kidney was not removed.

3) $N \rightarrow X$ The normal right kidney was transplanted into the hypertensive recipients. The infarcted kidney was removed. 


\section{Results}

When the infarcted kidneys were transplanted into the bilaterally nephrectomized recipients 1 or 2 weeks after ligating the renal arterial branch, the rats became hypertensive. One of the 6 rats which received the kidney infarcted 2 weeks before died probably because of hypertensive cardiovascular disease. The kidney 4 weeks after infarction from the hypertensive donor, however, failed to produce significant rise in BP of all 4 recipients (Protocol 1 , Fig. 1). When the normal kidneys were transplanted into the hypertensive

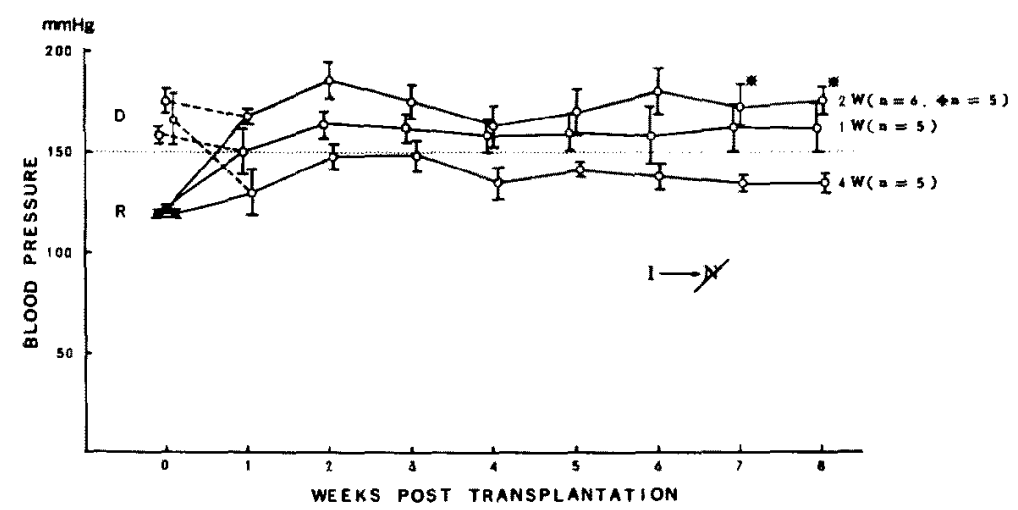

Fig. 1. Blood pressure of the bilaterally nephrectomized recipients after the transplantation of the kidney $(O)$ which had been infarcted 1,2, and 4 weeks before (Protocol 1). Circles at the top of interrupted lines are the BP of donors. Solid circles (O) indicate the $\mathrm{BP}$ of recipients at the time of transplantation. Means $\pm S E M$.

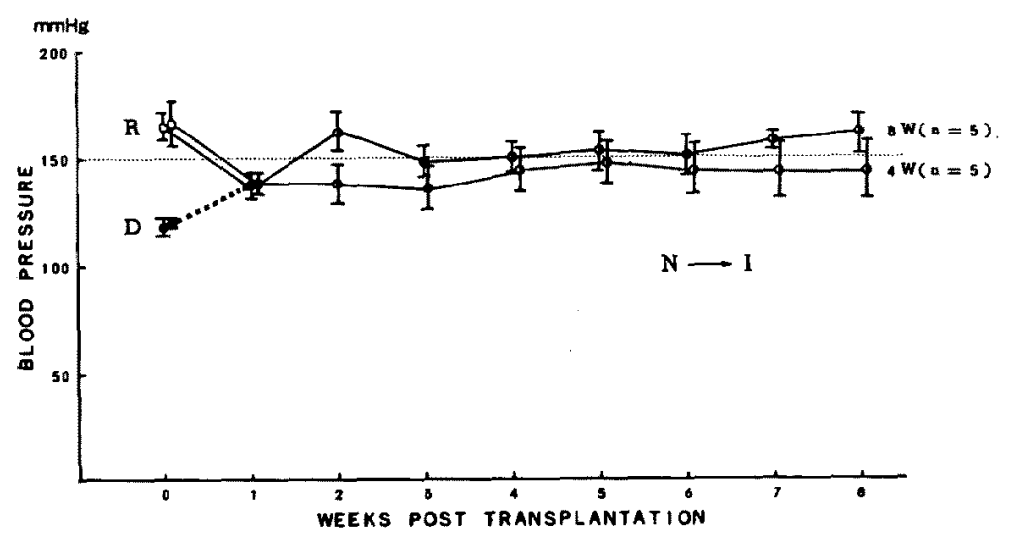

Fig. 2. Blood pressure of hypertensive recipients after the transplantation of the normal kidney without removing the infarcted kidneys (O) (Protocol 2). Operations were performed 4 and 8 weeks after the production of renal infarction. Means $\pm S E M$. 
rats 8 weeks after infarction, leaving the infarcted kidney in situ, BP remained high except 1 of 5 rats. When the transplantation was performed 4 weeks after infarction, BP stayed within a borderline level (Protocol 2, Fig. 2). In

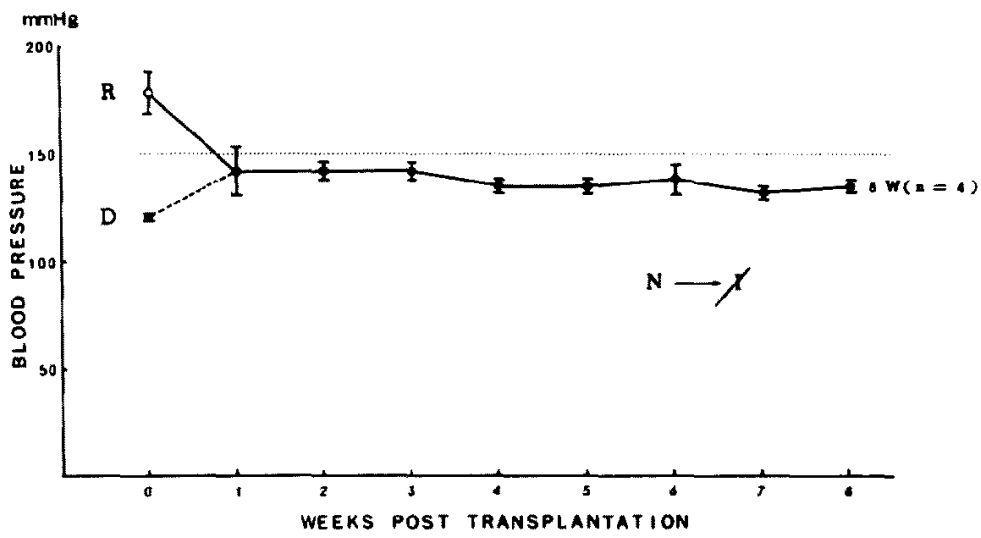

Fig. 3. Blood pressure of hypertensive recipients after the transplantation of the normal kidney with removing infarcted kidney produced 8 weeks before (O) (Protocol 3). Means \pm SEM.

Table I. PRA and KRA in Hypertensive Rats After Unilateral Renal Infarction and Contralateral Nephrectomy

\begin{tabular}{c|c|c}
\hline $\begin{array}{c}\text { Duration of Infarction } \\
\text { (wks) }\end{array}$ & $\begin{array}{c}\text { PRA } \\
\left(\mathrm{K} \times 10^{-5}\right)\end{array}$ & $\begin{array}{c}\text { KRA } \\
\left(\mathrm{K} \times 10^{-2}\right)\end{array}$ \\
\hline normal control & $25.0 \pm 14.1$ & $80.4 \pm 15.4$ \\
1 & $59.4 \pm 33.1$ & $156.8 \pm 33.5$ \\
2 & $6.7 \pm 1.3$ & $119.8 \pm 25.7$ \\
4 & $11.6 \pm 3.6$ & $82.2 \pm 12.8$ \\
8 & $9.8 \pm 2.2$ & $59.4 \pm 6.1$
\end{tabular}

Figures are means \pm SEM. $\quad(\mathrm{N}=5)$

Table II. PRA and KRA 8 Weeks After Renal Transplantation

\begin{tabular}{|c|c|c|c|c|c|}
\hline Protocol & $\begin{array}{c}\text { Duration of } \\
\text { Infarction (wks) }\end{array}$ & $\begin{array}{c}\text { No. of } \\
\text { Determination }\end{array}$ & $\mathrm{BP} *$ & $\begin{array}{c}\text { PRA } \\
\left(\mathrm{K} \times 10^{-5}\right)\end{array}$ & $\underset{\left(\mathrm{K} \times 10^{-2}\right)}{\mathrm{KRA}}$ \\
\hline \multirow{3}{*}{$I(I \rightarrow N)$} & 1 & 5 & $\mathrm{H}$ & $17.3 \pm 8.4$ & $89.7 \pm 41.3$ \\
\hline & 2 & 5 & $\mathrm{H}$ & $4.0 \pm 1.1$ & $45.7 \pm 3.3$ \\
\hline & 4 & 4 & $\mathrm{~N}$ & $11.4 \pm 4.9$ & $57.4 \pm 17.0$ \\
\hline \multirow{2}{*}{$2(N \rightarrow I)$} & 4 & 4 & $\mathrm{~N}-\mathrm{H}$ & $7.2 \pm 2.7$ & $\left\{\begin{array}{l}34.2 \pm 6.4(\mathrm{~N}) \\
86.4 \pm 24.6(\mathrm{I})\end{array}\right.$ \\
\hline & 8 & 6 & $\mathrm{H}$ & $11.3 \pm 2.7$ & $\left\{\begin{array}{l}35.8 \pm 8.5(\mathrm{~N}) \\
80.4 \pm 15.4(\mathrm{I})\end{array}\right.$ \\
\hline $3(\mathrm{~N} \rightarrow \mathrm{X})$ & 8 & 4 & $\mathrm{~N}$ & $5.7 \pm 1.4$ & $40.8 \pm 4.9$ \\
\hline
\end{tabular}

* Level of blood pressure: $\mathrm{N}$ : normotensive; $\mathrm{H}$ : hypertensive; $\mathrm{N}-\mathrm{H}$ : borderline ; $(\mathrm{N})$ : normal transplanted kidney; (I) : infarcted kidney. (Means \pm MES) 
5 hypertensive rats 8 weeks after infarction, BP was reduced to normal by the transplantation of the normal kidney and nephrectomy of the infarcted kidney (Protocol 3, Fig. 3).

PRA was significantly incrcased 1 wcek after infarction and contralateral nephrectomy. PRA returned to normal after 2 weeks. The same tendency was seen in KRA (Table I). Table II shows the PRA and KRA in transplanted rats 8 weeks after surgery. BP level and renin activity in plasma and kidney were not correlated.

\section{Discussion}

Present data confirmed essentially the results reported previously. ${ }^{1)}$ The infarcted kidney is involved in the development and maintenance of hypertension. Since the infarcted kidney which was allowed to remain in the donor over 4 weeks failed to elevate BP of recipients, some other factor than pressor mechanism must be operating in the hypertensive rats with renal infarction. This factor may be considered as maintenance mechanism because the presence of infarcted kidney is essential to maintain the blood pressure level after transplantation of the normal kidney to hypertensive recipients. The pressor mechanism terminates by 4 weeks after infarction, while the maintenance mechanism develops about 4 weeks after infarction. And the BP is expressed as the summation of the 2 mechanisms (Fig. 4). The normal kideny has a function which normalizes high blood pressure, but this mechanism does not compete with the maintenance mechanism of the infarcted kidney.

Previous study suggested that pressor mechanism was effective as long as 12 days, but present study demonstrated that pressor potency lasted more than

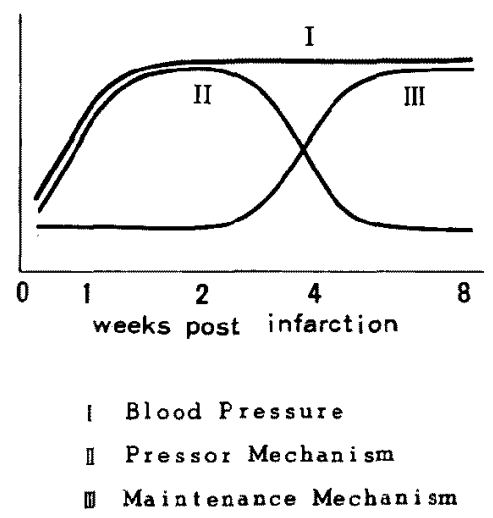

Fig. 4. Schematic expression of the possible role of kidney in hypertension due to renal segmental infarction. For details see the text. 
2 weeks and terminated by 4 weeks. This discrepancy in pressor persistency is not crucial, but probably due to the difference in rat strain, distribution of infarcted area, or laboratory conditions.

Bianchi et $\mathrm{a}^{\mathbf{6}}$ ) showed that normotensive rats developed hypertension after transplantation of the kidneys of spontaneously hypertensive rats (SHR), while BP of SHR normalized when the kidney from normotensive rat was transplanted into SHR. The same results were obtained when the transplantation was made at 3-4 months of age or young prehypertensive stage. Dahl, Heine, and Thompson" showed that resistant rat became prone to hypertension, when the kidney from the hypertensive-prone rat was transplanted into the hypertensive-resistant rat, while the sensitive rat became resistant to hypertension when the kidney from the resistant rat was transplanted. Differences by the age of rats at transplantation has not been reported. These results indicate that genetic influences to BP-level are determined predictably by the kidney, but existence of the 2 mechanisms in the kidney was not observed.

The present experiments do not provide any data suggestive to explain the pressor or maintenance mechanism of the infarcted kidney and the mechanism in the normal kidney which normalizes high blood pressure when the infarcted kidney had been removed. From the results on PRA and KRA, the renin-angiotensin system does not participate in either mechanism. Since transplanted kidney lacks neural innervation, these mechanisms may be operated by a humoral substance or excretory function of the kidney.

\section{REFERENCE}

1. Kawabe K, Merrill JP, Oken DE: Renal transplantation study of infarcted kidney as carriers of a hypertensive factor in the rat. Jap Heart J 13: 214, 1972

2. Fisher $B$, Lee $S$ : Microvascular surgical techniques in research with special reference to renal transplantation in the rat. Surgery 58:904, 1965

3. Lee S: An improved technique of renal transplantation in the rat. Surgery 61:771, 1967

4. Boucher R, Menard J, Genest J: A micromethod for measurement of renin in the plasma and kidney of rats. Canad J Physiol Pharmacol 45: 881, 1967

5. Nishimura $\mathrm{H}$, Sokabe $\mathrm{H}$ : Inhibition of angiotensinase in the rat plasma and kidney. Jap Heart J 9: 494, 1968

6. Bianchi G, Fox U, DiFrancesco GF, Giovanetti AM, Pagetti D: Blood pressure changes produced by kidney cross-transplantation between spontancously hypcrtensive rats and normotensive rats. Clin Sci Molec Med 47: 435, 1974

7. Dahl LK, Heine M, Thompson K: Genetic influence of the kidneys on blood pressure. Evidence from chronic renal homografts with opposite predispositions to hypertension. Circulat Res 34: 94, 1974 